\title{
Efectos de un programa de entrenamiento presencial vs prescripción a través de una aplicación móvil en personas mayores \\ Effects of face-to-face training intervention versus mobile application prescription among elderly \\ Adrià Muntaner Mas, Pere Palou Sampol, Josep Vidal Conti \\ Universidad de las Islas Baleares (España)
}

Resumen. El nivel de condición física es un marcador de salud para las personas mayores. El objetivo de este estudio fue evaluar y comparar la efectividad de un programa de ejercicio físico, administrado de forma presencial y dirigido versus la prescripción a través de una aplicación móvil. Veintitrés participantes de 55 a 73 años fueron incluidos en la intervención de 10 semanas de duración. Los participantes fueron distribuidos en un grupo presencial ( $\mathrm{n}=16)$ y en un grupo móvil (n=7). El grupo presencial recibió 20 sesiones (70-80 minutos de duración) a razón de dos semanales de actividad física centrada en ejercicio aeróbico y resistencia muscular, el grupo móvil recibió el mismo programa pero los contenidos fueron prescritos a través de una aplicación móvil. Presión arterial media, sistólica, diastólica, frecuencia cardiaca en reposo, porcentaje de masa muscular y de grasa corporal, perímetro de cintura, equilibrio, fuerza y capacidad aeróbica fueron evaluados antes y después de la intervención. El grupo presencial obtuvo mejoras en la presión arterial media, sistólica y diastólica, en la frecuencia cardiaca después del test y en la capacidad aeróbica $(p<.001, p<.001, p=.001, p=.003$, $p=.004$, respectivamente). No se observaron diferencias significativas en ninguna de las variables en el grupo móvil. Los resultados sugieren que el ejercicio dirigido es más efectivo en las variables analizadas que el prescrito mediante la aplicación móvil. Futuros estudios que impliquen muestras de mayor tamaño deben confirmar o refutar estos resultados.

Palabras clave. actividad física, telefonía móvil, entrenamiento de fuerza, ejercicio aeróbico, condición física, evaluación, Whatsapp.

Abstract. Physical fitness level is a health marker in elderly people. The aim of our study was to evaluate and compare the effectiveness of a physical exercise intervention, delivered face-to-face versus prescription through a mobile application. Twenty three participants (55-73 years old) were included in the 10-week intervention. Participants were allocated to the training group ( $\mathrm{n}=16$ ) or mobile group ( $\mathrm{n}=7$ ). The training group received 20 sessions (70-80 minutes per day) of physical exercise and were assigned to aerobic and resistance training (two sessions/per week). Intervention content was the same in the mobile group; only the delivery mode differed by means of a mobile application. We measured mean arterial pressure, systolic blood pressure, diastolic blood pressure, heart rate, percentage of muscle mass and body fat, balance, waist circumference, strength and aerobic capacity before and after 10 weeks of exercise intervention. The training group obtained improvements in mean arterial pressure, systolic blood pressure, diastolic blood pressure, heart rate after exercise and aerobic capacity $(p<.001, p<.001, p=.001, p=.003, p=.004$, respectively). There were no significant changes in the mobile group. The results suggest that face-to-face exercise is more effective than prescribed using mobile applications. Future studies involving larger sample sizes should confirm or refute these findings.

Keywords. physical activity, cell phones, resistance training, aerobic exercise, physical fitness, assessment, Whatsapp.

\section{Introducción}

Las repercusiones sobre la salud provocadas por la actividad física están ampliamente documentadas (Salinas, Cocca, Mohamed, \& Viciana, 2010). Peseal conocimiento de estos beneficios la inactividad física es la cuarta causa de muerte en el mundo (Kohl et al., 2012). En el estudio realizado por Guthold, Ono, Strong, Chatterji \& Morabia (2008) se analizó la prevalencia de inactividad física en 51 países, las conclusiones del estudio revelaron que el $17.7 \%$ de los participantes incluidos fueron insuficientemente activos. Estos resultados fueron similares independientemente del género analizado, además se observó que las personas más mayores fueron más inactivas físicamente, esta tendenciaincrementó con la edad de los participantes. Recientemente Sun, Norman \& While (2013) realizaron una revisión sistemática sobre la prevalencia de actividad física en personas mayores aportando que el porcentaje de adultos que cumplían los niveles de actividad física recomendados por la Organización Mundial de la Salud (OMS) variaba entre 2.4\% y 83\%, concentrándose la mayoría de estudios entre el 20-60\% de la muestra que conseguía cumplir las recomendaciones. En España, la población mayor de 15 años es insuficientemente activa (hombres $47.7 \%$ y mujeres 56.3\%), según las estimaciones de la OMS (2008). Los datos nacionales de 2011-2012 muestran que el 41.3\% de la población adulta (>15 años) fueron sedentarios (hombres 35.9\% y mujeres 46.6\%) (Instituto Nacional de Estadística, 2012).

Según una revisión reciente de la prestigiosa revista «The Lancet» se afirma que los avances en medicina y en la salud pública, la mejora en los niveles de vida y la reducción de la fertilidad han contribuido a la reducción de la mortalidad en muchas regiones del mundo (Mathers, Stevens, Boerma, White, \& Tobias, 2014). Este estudio concluye afirmando que en los países con altos ingresos, la esperanza de vida a la edad de 60 años ha aumentado en las últimas décadas. Las mujeres

Fecha recepción: 05-01-15- Fecha envío revisores: 05-01-15- Fecha de aceptación: 24-05-15 Adrià Muntaner Mas adria.muntaner@uib.es españolas tienen una esperanza de vida al nacer de 85.21 años y los varones de 79.3 años, estos valores se encuentran entre los más altos de la Unión Europea (García, Vilches \& Pujol, 2014).

Debido a la inactividad física y al aumento de la esperanza de vida, existe la necesidad de prestar atención al desarrollo de intervenciones más eficaces y que puedan llegar a un gran número de personas a bajo coste (Blackman et al., 2013). La efectividad de las intervenciones de actividad física presenciales (Richards, Hillsdon, Thorogood, y Foster, 2013) y no presenciales (Müller \& Khoo, 2014) ha sido estudiada encarecidamente, aún así parece ser que no hay unanimidad respecto a determinar cuál es más efectiva (Richards, Thorogood, Hillsdon, \& Foster, 2013). En este sentido la tecnología móvil se póstula como una alternativa eficaz a esta problemática puesto que su uso está en aumento en adultos. La Salud Móvil (mHealth) se ha convertido en un importante campo para la gestión de las enfermedades, la evaluación de las conductas saludables y para las intervenciones sobre comportamientos saludables. Las tecnologías móviles se están utilizando para estos fines puesto que ofrecen nuevos enfoques para las mediciones y nuevas alternativas para diseñar programas de intervención. Este crecimiento de la tecnología móvil ha llevado al desarrollo de numerosas intervenciones que han quedado recogidas en diversas revisiones sistemáticas (BortRoig, Gilson, Puig-Ribera, Contreras \& Trost, 2014; Fanning, Mullen \& McAuley, 2012; Stephens \& Allen, 2013). El uso de teléfonos móviles para la administración de intervenciones de actividad física resulta atractivo para los investigadores por varias razones: (1) existe un aumento marcado del uso del teléfono móvil, (2) por la evolución constante de sus capacidades técnicas, (3) por la tendencia habitual de las personas a llevar el teléfono móvil a cualquier lugar y (4) el apego de las personas a sus teléfono móviles (Klasnja y Pratt, 2012).

En este contexto las aplicaciones móviles están ganando popularidad entre los profesionales (Buijink, Visser, \& Marshall, 2013). Son muchas las ventajas del uso de Internet como herramienta incorporada a las aplicaciones móviles: accesibilidad constante, ajustes a las necesidades del usuario, capacidad de proporcionar retroalimentación individualizada, alcance masivo y características interactivas. Muchos 
investigadores y empresas comerciales han aprovechado estas características para diseñar diferentes tipos de aplicaciones orientadas a la promoción de hábitos saludables: aplicaciones que permiten registrar información sobre los hábitos alimenticios (Rollo, Ash, Lyons-Wall, \& Russell, 2011), aplicaciones que permiten registrar niveles de glucosa (Kim et al., 2014), aplicaciones que permiten registrar niveles de actividad física (Kirwan, Duncan, Vandelanotte, \& Mummery, 2012), aplicaciones que permiten monitorizar a pacientes con insuficiencia cardiaca (Seto et al., 2010) e hipertensión arterial (Logan et al., 2007), aplicaciones que ayudan a dejar de fumar(Buller, Borland, Bettinghaus, Shane, \& Zimmerman, 2014), a autogestionar el cuidado dela enfermedad pulmonar obstructiva crónica (Williams, Price, Hardinge, Tarassenko, \& Farmer, 2014) y aplicaciones que promueven la actividad física (van Dantzig, Geleijnse, \& van Halteren, 2012).

Las aplicaciones móviles son herramientas relativamentenuevas en la administración de intervenciones sobre actividad física, hecho constatable en el número de manuscritos publicados hasta la fecha. No obstante las aplicaciones móviles que promueven esta práctica son muy populares en las plataformas principales: de las 875.683 aplicaciones activas y disponibles en iTunes y las 696.527 en Google Play, 34.490 y 17.756 se catalogaron en el ámbito de Salud y Fitness (Middelweerd, Mollee, van der Wal, Brug, \& Te Velde, 2014). Muchas de estas aplicaciones suelen complementarse con alguna red social, de hecho hay un aumento continuo del uso de las redes sociales a nivel mundial. Las aplicaciones móviles basadas en redes sociales, como Whatsapp o Facebook, poseen una serie de características que podrían mejorar las intervenciones de apoyo social, incluyendo la capacidad de los usuarios para compartir información personal y mostrarla en tiempo real. Esta combinación de alcance y funcionalidad hace que las redes sociales sean un medio potencialmente eficaz para la ejecución de intervenciones de apoyo social (Cavallo et al., 2012). Los avances en la tecnología móvil significan que las aplicaciones móviles serán de vital importancia en un futuro cercano en el desarrollo de las intervenciones de actividad física. Pero mientras este rápido desarrollo está captando la atención de los usuarios existe una creciente preocupación por los peligros potenciales relacionados con el uso de las aplicaciones móviles para este uso (Buijink etal., 2013). Las aplicaciones móviles que promocionen el ejercicio físico deberían regularse y ser científicamente sólidas con el fin de garantizar su validez. Los objetivos del presente estudio fueron: (1) comprobar si el efecto del entrenamiento presencial es similar a la prescripción de ejercicio físico a través de una aplicación móvil, en relación con la condición física, parámetros cardiovasculares y antropométricos de las personas mayores y (2) evaluar la viabilidad de una intervención de ejercicio físico prescrita a través de una aplicación móvil basada en una red social.

\section{Metodología}

\section{Diseño}

El estudio fue un ensayo controlado sobre dos grupos experimentales: grupo presencial (GP) y grupo móvil (GM). La asignación a los grupos experimentales fue por conveniencia; siete de ellos fueron asignados al grupo móvil y 16 al grupo presencial. Seleccionamos a los participantes utilizando diferentes técnicas entre las cuales se incluyeron: información cara a cara, distribuciones de pósters informativos; con el intento de aglutinar una muestra heterogénea. Inicialmente se realizó una selección de los participantes elegibles y se excluyó a aquellos que no cumplían con los criterios de inclusión, posteriormente los participantes fueron asignados a los grupos experimentales. Antes de iniciar los programas de ejercicio físico el equipo investigador realizó una reunión informativa y explicó por separado a cada uno de los grupos el procedimiento a seguir durante la intervención, en esta reunión se entregó el consentimiento informado.

\section{Participantes}

La muestra estuvo formada por 23 personas mayores de edades comprendidas entre 55 y 73 años. Los criterios de inclusión fueron: tener 55 años o más, tener móvil propio y acceso a internet, estar clínicamente estable, ser capaz de realizar actividad física y tener capacidad para afrontar las pruebas de evaluación. Los criterios de exclusión fueron: padecer una enfermedad aguda o crónica, tener condiciones médicas u otro problema físico que necesitara una especial atención para realizar actividad física (historial de fractura de alguna extremidad superior o inferior, demencia, problemas psicológicos, enfermedad neuromuscular, historial de infarto de miocardio, osteoporosis, cáncer y/o diabetes). El estudio siguió las normas deontológicas reconocidas por la Declaración de Helsinki (revisión de Seúl, octubre 2008). El estudio también fue sometido a valoración y obtuvo la aprobación del Comité de Ética de la Investigación de la Universidad de las Islas Baleares (CEI-IB) y fue registrado en ClinicalTrials.gov (NCT02327975).

\section{Intervención}

Los programas de entrenamiento se llevaron a cabo del 21 de Enero del 2013 al 28 de Marzo del 2013. La intervención transcurrió durante 10 semanas, a razón de dos sesiones semanales de 70-80 minutos de duración acumulando un total de 20 sesiones. Ambos grupos experimentales recibieron el mismo programa de ejercicio físico, basado en fortalecimiento muscular y ejercicio aeróbico. Las sesiones se estructuraron en cuatro partes: fase de calentamiento, fase de fuerza, fase de capacidad aeróbica y fase de vuelta a la calma. En la fase de calentamiento, con una duración de 10 minutos, se realizó carrera continua y ejercicios de movilidad articular. En la fase de fuerza, con una duración de 25 minutos, se realizaron ejercicios de fortalecimiento muscular; el equipo utilizado para las actividades de esta fase fueron las bandas elásticas Thera-Band® ${ }^{\circledR}$. En la fase de capacidad aeróbica, con una duración de 25-35 minutos, se realizó carrera continua. La fase de vuelta a la calma consistió en la realización de estiramientos de los músculos principales y tuvo una duración de 10 minutos.

\section{Grupo presencial}

Este grupo experimental recibió la intervención de forma presencial y monitorizada bajo la supervisión directa de un miembro del grupo de investigación para asegurar la correcta técnica, seguridad y la adecuada intensidad del ejercicio. La intensidad de la resistencia de las bandas elásticas se calculó mediante el número de repeticiones y la «OMNI Resistance Scale» de Colado et al. (2012). La parte principal de las sesiones consistió en la realización de los siguientes ejercicios: press de pectoral, press de hombro, elevaciones laterales, remo sentado, polea al pecho, curl de bíceps, extensión de codo con mancuerna, curl femoral, extensión de rodilla, sentadilla, lunge y contracción abdominal; con incidencia sobre los grupos musculares: dorsal, pectoral, deltoides, bíceps, tríceps, cuádriceps e isquiotibiales. El programa de entrenamiento se dividió en dos mesociclos de 5 semanas para optimizar las ganancias de fuerza e hipertrofia muscular. El objetivo del primer mesociclo (semanas 1-5) consistió en la adaptación de los participantes a la intensidad y a los ejercicios prescritos (intensidad de 6-7 en la OMNI Resistance Scale) y un gran número de repeticiones (15-20) de cuatro a cinco series, con periodos de descanso de entre 90 segundos entre series y 120 segundos entre ejercicios. El segundo mesociclo (semanas 6-10) fue diseñado para producir hipertrofia muscular y la intensidad fue de 8-9 en la OMNI Resistance Scale, con 10-15 repeticiones, entre tres y cuatro series, con periodos de descanso de 45 segundos entre series y 90 segundos entre ejercicios. El número de repeticiones varió durante los mesociclos, empezando por 20 durante las semanas 1-2, 15 durante las semanas 3-5, 10 durante las semanas 6-7 y 15 durante las semanas 8-10. Cada vez que el número de repeticiones cambió la resistencia utilizada en la banda elástica también lo hizo. Por ejemplo si el número de repeticiones disminuía la resistencia de la banda elástica incrementaba. El ejercicio aeróbico se llevó a cabo siguiendo las recomendaciones del American College of Sport Medicine (2007) para las personas mayores y consistió en la realización de carrera continua durante 25-35 minutos variando entre el 60-80\% de la frecuencia cardiaca máxima durante los dos días. Durante las semanas 1-3 los participantes realizaron 25 minutos al 60\% de la frecuencia cardiaca máxima, durante las semanas 4-6 
realizaron 30 minutos al 70\% de la frecuencia cardiaca máxima y entre las semanas 7-10 realizaron 35 minutos al 80\% de la frecuencia cardiaca máxima.

\section{Grupo móvil}

Los contenidos sobre ejercicio físico que recibieron estos participantes fueron los mismos que el grupo presencial, con la única y principal diferencia en la forma de transmisión de la información. Siguiendo el ejemplo de Stuckey et al. (2011) se utilizó la telefonía móvil, concretamente una aplicación móvil de índole social (Whatsapp) para transmitir el programa de ejercicio físico. Los participantes de este grupo recibieron dos vídeos (archivo digital multimedia disponible para su descarga en un reproductor multimedia) por semana durante las 10 semanas de entrenamiento, cada vídeo tuvo una duración de 5 min. Los archivos eran descargados a través de la aplicación móvil quedando almacenados en los dispositivos móviles. Los participantes de este grupo realizaron las dos sesiones semanales en días no consecutivos y de forma autónoma, los días de la semana del entrenamiento fueron elegidos por los propios participantes.

\section{Variables e instrumentos de medida}

Se evaluaron a los dos grupos experimentales en dos momentos distintos: antes del inicio de la intervención (pretest) y después (posttest). Cada período de medición consistió en la realización completa de la batería de pruebas. La información de fondo recopilada incluyó edad, género, peso, talla, índice de masa corporal (IMC) y frecuencia cardiaca.

Para la medición de la fuerza se utilizó el test de fuerza máxima de prensión manual. La fuerza de prensión manual fue medida con un dinamómetro analógico (T.K.K. 5001 grip-A; Takey, Tokyo, Japan) y los resultados se anotaron en kilogramos. Al realizar la medición, los participantes fueron instruidos para mantener la posición bípeda estándar durante toda la prueba y con el brazo en completa extensión. Cada sujeto realizó (alternativamente con ambas manos) dos veces la prueba, permitiendo un descanso de 30-60 segundos entre mediciones. La capacidad aeróbica fue medida mediante el 2-minute step test (Rikli \& Jones, 2012). La realización del test consiste en marchar en el sitio el mayor número de veces que sea posible en 2 minutos. El control de la frecuencia cardíaca durante el programa de entrenamiento fue medido mediante el pulsómetro (Polar FT1). La medición del equilibrio estático se llevó a cabo mediante el test de flamenco (Committee of Experts on Sport Research, 1988). Para la medición de la frecuencia cardíaca en reposo, de la presión arterial sistólica y diastólica se utilizó el dispositivo The Omron Elite 7300W (Omron Healthcare Inc, Bannockburn, IL, USA). La altura se midió utilizando un tallímetro portátil (Seca 217). El perímetro de cintura se midió con una cinta métrica flexible (Lufkin W606PM) colocada alrededor de la cintura del participante exactamente a la altura de su ombligo. Se midió el peso, la grasa corporal, la masa muscular y el IMC mediante la utilización de una báscula de bioimpedancia (Omron HBF-500 Body Composition Monitor with Scale). La presión arterial media fue calculada como la presión arterial diastólica más un tercio de la presión arterial sistólica menos la presión arterial diastólica (Zheng et al., 2008). El ratio cintura-talla fue calculado como la división de la circunferencia de cintura entre la talla.

\section{Análisis estadístico}

Los datos fueron analizados con el programa estadístico SPSS (v.19.0 de SPSS Inc., Chicago, IL, EE.UU). Realizamos una estadística descriptiva en la que calculamos las medias y las desviaciones típicas de todas las variables. La normalidad de las variables fue determinada con la prueba de Shapiro-Wilk, dado que las condiciones se cumplieron llevamos a cabo pruebas paramétricas. Las diferencias entre las medias de todas las variables desde la medición inicial has- ta la décima semana fueron comparadas mediante el $t$-test pareado, con la distribución de Student. Se estableció un nivel de significación d» .05 para todos los análisis. Puesto que el tamaño de los grupos fue pequeño se procedió a calcular la $d$ de Cohen para conocer el tamaño del efecto mediante la siguiente fórmula (Cohen, 1988):

$$
d=\frac{M_{1}-M_{2}}{\sqrt{\frac{\sigma_{1}^{2}+\sigma_{2}^{2}}{2}}}
$$

El tamaño del efecto se considera pequeño para un valor de $d$ en torno a 2 mediano para un valor de $d$ en torno a .5 y grande para un valor de $d$ en torno a .8 o superior.

\begin{tabular}{|c|c|c|c|c|c|}
\hline & \multicolumn{2}{|c|}{ Grupo presencial (n=16) } & \multicolumn{2}{|c|}{ Grupo móvil (n=7) } & \multirow[b]{2}{*}{ Valor-p } \\
\hline & Media & DE & Media & DE & \\
\hline Edad (años) & 64.00 & 5.44 & 62.86 & 5.11 & $.637^{\circ}$ \\
\hline Peso $(\mathrm{kg})$ & 63.75 & 11.56 & 68.61 & 8.93 & .335 \\
\hline Talla $(\mathrm{cm})$ & 161.53 & 5.83 & 167.14 & 7.22 & .101 \\
\hline $\operatorname{IMC}(\mathrm{kg} / \mathrm{m} 2)$ & 24.42 & 4.24 & 24.57 & 2.94 & .934 \\
\hline FC en reposo (ppm) & 71.25 & 10.89 & 70.57 & 7.23 & .882 \\
\hline
\end{tabular}

\section{Resultados}

En la Tabla 1 se presentan las medias y desviaciones típicas de las variables obtenidas por el grupo presencial y el grupo móvil, en el momento anterior a iniciar los programas y al finalizar el proceso de intervención(después de 10 semanas). Observándose que la media de la edad de los participantes fue de $63.65 \pm 5.25$ y el IMC medio fue de $24.47 \pm 3.82$.

Las Tablas 2 y 3 muestran el efecto de las intervenciones en los diferentes componentes analizados: parámetros cardiovasculares, antropométricos y de condición física, respectivamente. Los datos sugieren que se producen diferencias en el grupo presencial y estas son significativas en las variables: presión arterial sistólica, diastólica y media, frecuencia cardíaca después de la realización del test de capacidad aeróbica y en el 2-minute step test $\left(t_{1.75}=5.86 ; p=.000, t_{1.75}=3.96 ; p\right.$ $\left.=.001, t_{1.75}=5.39 ; p=.000, t_{1.75}=3.52 ; p=.003, t_{1.75}=-3.43 ; p=.004\right)$. No alcanzaron la significación para este grupo las variables de condición física (excepto el 2-minute step test) y los valores antropométricos. Los resultados del grupo móvil no alcanzaron la significación en ninguna de las variables. Las diferencias en términos absolutos entre las medias, antes y después del entrenamiento, son ligeramente superiores en el grupo presencial en la mayoría de las variables analizadas, excepto en la frecuencia cardiaca en reposo ( $\mathrm{GP}=.50 ; \mathrm{GM}=4.42$ ), porcentaje de grasa corporal ( $\mathrm{GP}=.23 ; \mathrm{GM}=2.38$ ), perímetro de la cintura (GP = $.31 ; \mathrm{GM}=.85$ ) y la fuerza de prensión manual $(\mathrm{GP}=.06$; $\mathrm{GM}=2.28$ ). El grupo experimental presencial obtuvo valores más elevados del tamaño del efecto en la presión arterial sistólica ( $\mathrm{GP}=.52$; $\mathrm{GM}=.16$ ), diastólica (GP = .44; $\mathrm{GM}=-.18$ ) y media (GP = .51; $\mathrm{GM}=-.05$ ), frecuencia cardíaca después dela realización del test de capacidad aeróbica $(\mathrm{GP}=.39 ; \mathrm{GM}=.33$ ) y en el 2-minute step test $(\mathrm{GP}=-.40 ; \mathrm{GM}=$ $.09)$.

\begin{tabular}{|c|c|c|c|c|c|c|c|c|c|c|c|c|}
\hline \multirow[t]{3}{*}{ Variables } & \multicolumn{6}{|c|}{ Grupo presencial $(\mathrm{n}=16)$} & \multicolumn{6}{|c|}{ Grupo móvil (n=7) } \\
\hline & \multirow{2}{*}{$\begin{array}{c}\begin{array}{c}\text { Pre- } \\
\text { ejercicio }\end{array} \\
\text { Media (DE) }\end{array}$} & \multirow{2}{*}{$\begin{array}{c}\begin{array}{c}\text { Post- } \\
\text { ejercicio }\end{array} \\
\text { Media (DE) }\end{array}$} & \multicolumn{2}{|c|}{$95 \%$ IC } & \multirow[b]{2}{*}{ Post-Pre(TE) } & \multirow[b]{2}{*}{$p$} & \multirow{2}{*}{$\begin{array}{c}\begin{array}{c}\text { Pre- } \\
\text { ejercicio }\end{array} \\
M \\
\text { (DE) }\end{array}$} & \multirow{2}{*}{$\begin{array}{c}\begin{array}{c}\text { Post- } \\
\text { ejercicio }\end{array} \\
M \\
\text { (DE) }\end{array}$} & \multicolumn{2}{|c|}{$95 \%$ IC } & \multirow[b]{2}{*}{ Post-Pre (TE) } & \multirow[b]{2}{*}{$p$} \\
\hline & & & I & $\mathrm{s}$ & & & & & I & $\mathrm{s}$ & & \\
\hline $\begin{array}{l}\text { Presi ón artenial al } \\
\text { sis tólica (mmg) }\end{array}$ & $\begin{array}{l}145.63 \\
(12.15)\end{array}$ & $\begin{array}{l}129.13 \\
(14.74)\end{array}$ & 10.50 & 22.50 & $-16.50 \pm 11.26 b$ & .000 & $\begin{array}{l}131.43 \\
(15.05)\end{array}$ & $\begin{array}{l}126.29 \\
(15.07)\end{array}$ & -11.26 & 21.55 & $-5.14 \pm 17.74 a$ & .472 \\
\hline $\begin{array}{c}\text { Presión arterial } \\
\text { diastólica (mmHg) }\end{array}$ & $\begin{array}{l}86.44 \\
(6.87)\end{array}$ & $\begin{array}{l}77.75 \\
(10.47)\end{array}$ & 4.01 & 13.35 & $-8.68 \pm 8.76 b$ & .001 & $\begin{array}{l}78.00 \\
(12.79)\end{array}$ & $\begin{array}{l}83.00 \\
(14.05)\end{array}$ & -15.29 & 5.29 & $5.00 \pm 11.13 \mathrm{a}$ & .280 \\
\hline $\begin{array}{l}\text { Presión arterial media } \\
(\mathrm{mmHg})\end{array}$ & $\begin{array}{l}106.16 \\
(7.57)\end{array}$ & $\begin{array}{c}94.87 \\
(11.02)\end{array}$ & 6.80 & 15.78 & $-11.29 \pm 8.42 \mathrm{~b}$ & .000 & $\begin{array}{c}95.80 \\
(13.18)\end{array}$ & $\begin{array}{c}97.42 \\
(13.97)\end{array}$ & -13.65 & 10.41 & $1.61 \pm 13.01 \mathrm{a}$ & .753 \\
\hline FC en reposo & $\begin{array}{c}71.25 \\
(10.89)\end{array}$ & $\begin{array}{l}70.75 \\
(9.16)\end{array}$ & -4.60 & 5.60 & $-.50 \pm 9.58 \mathrm{a}$ & .838 & $\begin{array}{l}70.57 \\
(7.23)\end{array}$ & $\begin{array}{l}75.00 \\
(9.96)\end{array}$ & -9.46 & .60 & $4.42 \pm 5.44 \mathrm{~b}$ & .075 \\
\hline FC después del test & $\begin{array}{l}104.81 \\
(19.92)\end{array}$ & $\begin{array}{r}90.69 \\
(11.80)\end{array}$ & 5.58 & 22.67 & $-14.12 \pm 16.03 b$ & .003 & $\begin{array}{c}99.14 \\
(10.46)\end{array}$ & $\begin{array}{l}92.00 \\
(9.71)\end{array}$ & -.01 & 14.29 & $-7.14 \pm 7.73 \mathrm{~b}$ & .050 \\
\hline
\end{tabular}

IC: intervalo de confianza; I: inferior, S: superior. 
Efectos de la intervención en parámetros antropométricos y de condicón física.

\begin{tabular}{|c|c|c|c|c|c|c|c|c|c|c|c|c|}
\hline Variables & \multicolumn{6}{|c|}{ Grupo presencial (n=16) } & \multicolumn{6}{|c|}{ Grupo mó vil (n=7) } \\
\hline Masa muscular (\%) & $26.59(5.18)$ & $28.88(3.90)$ & -5.23 & .66 & $2.28 \pm 5.53 b$ & .119 & $32.55(4.59)$ & $31.48(3.79)$ & -.88 & 3.01 & $-1.07 \pm 2.09 a$ & .225 \\
\hline Grasa corporal (\%) & $32.00(7.60)$ & $32.23(8.85)$ & -2.74 & 2.26 & $.23 \pm 4.70 \mathrm{a}$ & .843 & $25.54(7.18)$ & $27.92(6.27)$ & -5.22 & .45 & $2.38 \pm 3.07 \mathrm{a}$ & .086 \\
\hline Ratio cintura-talla & $.55(.06)$ & $.55(.06)$ & -.007 & .003 & $.00 \pm .01 \mathrm{a}$ & .463 & $.54(.05)$ & $.54(.05)$ & -.01 & .00 & $.00 \pm .00 \mathrm{a}$ & .084 \\
\hline Fuerza de prensión manual (kg) & $26.50(7.67)$ & $26.56(7.57)$ & -1.44 & 1.32 & $.06 \pm 2.59 \mathrm{a}$ & .924 & $28.71(14.33)$ & $31.00(14.23)$ & -4.59 & .02 & $2.28 \pm 2.49 a$ & .052 \\
\hline Equilibrio (s) & $39.94(22.46)$ & $47.50(18.41)$ & -18.93 & 3.81 & $7.56 \pm 21.34 a$ & 177 & $41.86(23.11)$ & $39.71(19.47)$ & -9.89 & 14.18 & $-2.14 \pm 13.01 \mathrm{a}$ & .678 \\
\hline 2-minute step test & $122.13(43.46)$ & $156.25(32.88)$ & -55.27 & -12.97 & $34.12 \pm 39.69 \mathrm{~b}$ & .004 & $139.71(29.31)$ & $146.29(36.48)$ & -25.02 & 11.87 & $6.57 \pm 19.49 \mathrm{a}$ & .417 \\
\hline
\end{tabular}

\section{Discusión}

Los resultados observados en el presente estudio sugieren que una intervención de ejercicio físico dirigida presencialmente, basada en el fortalecimiento muscular y trabajo aeróbico, consigue mejoras muy significativas en ciertos factores de riesgo cardiovascular y en la capacidad aeróbica en personas mayores. Según nuestros datos la misma intervención pero administrada a través de una aplicación móvil no obtiene mejoras en ninguna de las variables analizadas. La intervención no obtuvo mejoras en parámetros antropométricos ni en algunos parámetros de condición física en ninguno de los grupos experimentales.

Muchos programas de ejercicio físico administrados de forma presencial han centrado su atención en la disminución de algunos factores de riesgo cardiovascular (Corrick, Hunter, Fisher, \& Glasser, 2013; Moreira, Cucato, Terra, \& Ritti-Dias, 2014) y en la capacidad aeróbica (Lamina, Okoye, \& Hanif, 2014; Severinsen, Jakobsen, Pedersen, Overgaard, \& Andersen, 2014), los resultados han sido similares a los nuestros. Después de un programa de 16 semanas de ejercicio aeróbico combinado con fortalecimiento muscular una vez por semana Corrick et al. (2013) observaron una disminución de 8.3 mmHg en la presión sistólica y de $4.3 \mathrm{mmHg}$ en la presión diastólica. En otra intervención basada en la realización de ejercicio aeróbico combinado con entrenamiento con sobrecarga durante 12 semanas Severinsen et al. (2014) encontraron mejoras significativas en la capacidad aeróbica, lo que confirma nuestros resultados del grupo presencial. Nuestros datos sugieren que el grupo móvil disminuye la presión sistólica después de las 10 semanas pero no de forma significativa, en cambio no se observan cambios relevantes en la presión diastólica. Joo y Kim, (2007) enviaron mensajes de texto a través de telefonía móvil, en ellos animaban a los participantes a aumentar sus niveles de actividad física, tras la intervención no se observó disminución significativa en la presión sistólica y diastólica. Stuckey, Kiviniemi, \& Petrella (2013) proporcionaron una Blackberry ${ }^{\circledR}$ Curve 8300 a cada uno de los participantes para monitorizar y prescribir ejercicio físico, sus resultados no mostraron cambios en la presión sistólica pero se observó una disminución importante en la presión diastólica.

En relación con los valores de peso, grasa corporal, masa muscular y perímetro de cintura se observa que los datos de la segunda toma son sensiblemente peores en ambos grupos experimentales. Nuestro estudio concuerda con los resultados de Binder et al. (2005) dónde sus datos muestran que no hay una disminución de la grasa corporal tras un programa de ejercicio físico. Levinger et al. (2008) tras realizar un entrenamiento de resistencia de forma presencial no obtuvieron mejoras en el perímetro de cintura, estos valores coinciden con nuestros resultados. Otra intervención utilizó el teléfono móvil para monitorizar el ejercicio prescrito a través de este dispositivo, en este estudio se observó una disminución del perímetro de cintura (Stuckey et al., 2011). Por otro lado nuestro grupo experimental móvil no muestra cambios en el perímetro de cintura, esto puede ser debido a las diferencias entre los participantes incluidos en los estudios.

Previos manuscritos han demostrado la validez del uso de las bandas elásticas para el incremento de fuerza en personas mayores, en intervenciones dirigidas de forma presencial (Cheung, Cinnadaio, Russo, \& Marek, 2009; Yasuda et al., 2014) como en programas prescritos en el hogar (Colado et al., 2010; Zion, De Meersman, Diamond \& Bloomfield, 2003). En nuestro estudio el $75 \%$ y el $85.7 \%$ de los parti- cipantes incrementaron la fuerza en el programa presencial y móvil respectivamente, no obstante no se alcanzó la significación. Esto puede ser debido a que la activación muscular depende del nivel de resistencia y del estiramiento de las bandas elásticas durante el ejercicio (Andersen et al., 2010). Wang et al. (2014) utilizaron la telefonía móvil para la prescripción de ejercicio físico en pacientes con enfermedad pulmonar obstructiva crónica, en este estudio se observó un aumento de la fuerza. Esta discordancia puede ser debido a la diferencia de duración entre los programas. Existe una evidencia débil de que el fortalecimiento muscular sea efectivo para la mejora del equilibrio en personas mayores (Howe, Rochester, Neil, Skelton \& Ballinger, 2011). Los datos indican que hubo un incremento de 7.56 ( $p=.177$ ) segundos en el grupo presencial, aunque no se alcanzó la significatividad. En esta misma línea existe coincidencia con el estudio de Yu, An \& Kang (2013) quienes tras un programa de fortalecimiento muscular con bandas elásticas no encontraron mejoras en personas mayores de 60 años. Martínez, Ortega, Párraga, \& Moreno (2010) obtuvieron mejoras en el equilibrio tras un programa de entrenamiento mediante videojuegos. La disparidad de los resultados podría ser explicada por la variedad de las tecnologías utilizadas en ambos estudios.

\section{Limitaciones y fortalezas del estudio}

La principal limitación del estudio es el tamaño de la muestra y su selección por conveniencia. Los resultados deberían ser interpretados con precaución hasta que ellos puedan ser verificados en estudios multicéntricos con muestras más grandes. Otra limitación, fueron las enormes diferencias en las distancias de estiramiento de las bandas elásticas en los ejercicios de fortalecimiento. En consecuencia hubo grandes diferencias individuales en la intensidad relativa del ejercicio. La evaluación de los parámetros antropométricos se realizó mediante una báscula de bioimpedancia (Omron HBF-500 Body Composition Monitor with Scale), antes y después de la intervención. Futuros estudios deberían utilizar métodos más objetivos, por ejemplo la densiometría ósea, lo que permitiría una evaluación más precisa de estos parámetros. En futuros estudios se debería incluir un grupo control y un grupo en el que se combinara el entrenamiento dirigido con la prescripción a través de una aplicación móvil.

Una de las fortalezas del estudio es la utilización de dos intervenciones de ejercicio físico diferentes, por esta razón el manuscrito tiene el potencial para hacer una importante contribución a la comprensión de las estrategias dirigidas al incremento de la salud en personas mayores con o sin componente de telefonía móvil. En nuestro estudio la aplicación móvil sirve para múltiples propósitos: proporcionar retroalimentación inmediata, administrar programas de ejercicio físico, apoyo social, proporcionar un modelo de cómo realizar los ejercicios. Según Conroy, Yang \& Maher, (2014) esta última característica está catalogada como la segunda mejor técnica de cambio de comportamiento en actividad física mediante aplicaciones móviles. Bajo nuestro mejor conocimiento, es la primera intervención en actividad física conducida mediante una aplicación móvil de índole social y que incorpore las funciones anteriormente mencionadas. También es el primero en utilizar un diseño de ensayo controlado para probar la eficacia de una intervención de este tipo en personas mayores. Adicionalmente destacar la innovación del uso de Whatsapp como medio de comunicación social para la implementación de la intervención y que ha permitido: mayor interacción entre participantes y equipo investigador, rápida 
disponibilidad de los participantes, aumento de la accesibilidad y apoyo emocional social. La aplicación de esta intervención podría ayudar a mejorar la salud y forma física de las personas mayores con el evidente potencial sobre la reducción de gastos económicos directos (asistencia sanitaria).

\section{Conclusiones}

Los resultados sugieren que una intervención de 10 semanas administrada de forma presencial y basada en ejercicio físico es más efectiva que la misma intervención prescrita a través de una aplicación móvil, en relación con parámetros cardiovasculares. Este estudio añade evidencia científica al uso de una aplicación móvil basada en una red social para la promoción de actividad física, dónde se demuestra la viabilidad y la posibilidad de aplicar este tipo de intervenciones en personas mayores, demostrando que esta herramienta no es exclusiva de los adolescentes y adultos jóvenes. El presente artículo también confirma la viabilidad del mismo, y realiza una aproximación que será útil para futuros investigadores que pretendan indagar en esta línea.

\section{Referencias}

Abellán García, A., Vilches Fuentes, J., Pujol Rodríguez, R. (2014). «Un perfil de las personas mayores en España, 2014. Indicadores estadísticos básicos». Madrid, Informes Envejecimiento en red $n^{\circ}$ 6.

Andersen, L. L., Andersen, C. H., Mortensen, O. S., Poulsen, O. M., Bjørnlund, I. B. T., \& Zebis, M. K. (2010). Muscle activation and perceived loading during rehabilitation exercises: comparison of dumbbells and elastic resistance. Physical Therapy, 90(4), 538-49. doi:10.2522/ptj.20090167

Binder, E. F., Yarasheski, K. E., Steger-May, K., Sinacore, D. R., Brown, M., Schechtman, K. B., \& Holloszy, J. O. (2005). Effects of progressive resistance training on body composition in frail older adults: results of a randomized, controlled trial. The Journals of Gerontology. Series A, Biological Sciences and Medical Sciences, 60(11), 1425-31.

Blackman, K. C., Zoellner, J., Berrey, L. M., Alexander, R., Fanning, J., Hill, J. L., \& Estabrooks, P. A. (2013). Assessing the internal and external validity of mobile health physical activity promotion interventions: a systematic literature review using the RE-AIM framework. Journal of Medical Internet Research, 15(10), e224. doi:10.2196/jmir.2745

Bort-Roig, J., Gilson, N. D., Puig-Ribera, A., Contreras, R. S., \& Trost, S. G. (2014). Measuring and influencing physical activity with smartphone technology: a systematic review. Sports Medicine (Auckland, N.Z.), 44(5), 671-86. doi:10.1007/s40279-014-0142-5

Buijink, A. W. G., Visser, B. J., \& Marshall, L. (2013). Medical apps for smartphones: lack of evidence undermines quality and safety. Evidence-Based Medicine, 18(3), 90-2. doi:10.1136/eb-2012100885

Buller, D. B., Borland, R., Bettinghaus, E. P., Shane, J.H., \& Zimmerman, D. E. (2014). Randomized trial of a smartphone mobile application compared to text messaging to support smoking cessation. Telemedicine Journal and E-Health: The Official Journal of the American Telemedicine Association, 20(3), 206-14. doi:10.1089/ tmj.2013.0169

Cavallo, D. N., Tate, D. F., Ries, A. V, Brown, J. D., DeVellis, R. F., \& Ammerman, A. S. (2012). A social media-based physical activity intervention: a randomized controlled trial. American Journal of Preventive Medicine, 43(5), 527-32. doi:10.1016/ j.amepre.2012.07.019

Cohen, J.(1988). Statistical power analysis for the behavioral sciences. Statistical Power Analysis for the Behavioral Sciences (Vol. 2nd). doi:10.1234/12345678

Colado, J. C., Garcia-Masso, X., Pellicer, M., Alakhdar, Y., Benavent, J., \& Cabeza-Ruiz, R. (2010). A comparison of elastic tubing and isotonic resistance exercises. International Journal of Sports Medicine, 31(11), 810-7. doi:10.1055/s-0030-1262808

Colado, J. C., Garcia-Masso, X., Triplett, T. N., Flandez, J., Borreani, S., \& Tella, V. (2012). Concurrent Validation of the OMNIResistance Exercise Scale of Perceived Exertion With Thera-Band Resistance Bands. Journal of Strength and Conditioning Research. doi:10.1519/JSC.0b013e318245c0c9

Conroy, D. E., Yang, C.-H., \& Maher, J. P. (2014). Behavior change techniques in top-ranked mobile apps for physical activity. American Journal of Preventive Medicine, 46(6), 649-52. doi:10.1016/j.amepre.2014.01.010

Cornelissen, V. A., Verheyden, B., Aubert, A. E., \& Fagard, R. H. (2010). Effects of aerobic training intensity on resting, exercise and post-exercise blood pressure, heart rate and heart-rate variability. Journal of Human Hypertension, 24(3), 175-82. doi:10.1038/ jhh.2009.51

Corrick, K. L., Hunter, G. R., Fisher, G., \& Glasser, S. P. (2013). Changes in vascular hemodynamics in older women following 16 weeks of combined aerobic and resistance training. Journal of Clinical Hypertension (Greenwich, Conn.), 15(4), 241-6. doi:10.1111/ jch.12050

Cheung, N. W., Cinnadaio, N., Russo, M., \& Marek, S. (2009). A pilot randomised controlled trial of resistance exercise bands in the management of sedentary subjects with type 2 diabetes. Diabetes Research and Clinical Practice, 83(3), e68-71. doi:10.1016/ j.diabres.2008.12.009

Encuesta Nacional de Salud de España. (2012). Madrid, Ministerio de Sanidad, Servicios Sociales e Igualdad. Instituto Nacional de Estadística, 2012 (http://www.msc.es/estadEstudios/estadisticas/ en cue st a N a c i on a l / encuest a N a c 2011 / DeterminantesSalud_DistribucionPorcentual.pdf, acceso 4 deEnero de 2015).

Fanning, J., Mullen, S. P., \& McAuley, E. (2012). Increasing physical activity with mobile devices: a meta-analysis. Journal of Medical Internet Research, 14(6), e161. doi:10.2196/jmir.2171

Guthold, R., Ono, T., Strong, K. L., Chatterji, S., \& Morabia, A. (2008). Worldwide Variability in Physical Inactivity. A51-Country Survey. American Journal of Preventive Medicine,34,486-494. doi:10.1016/ j.amepre.2008.02.013

Howe, T. E., Rochester, L., Neil, F., Skelton, D. A., \& Ballinger, C. (2011). Exercise for improving balance in older people. The Cochrane Database of Systematic Reviews, (11), CD004963. doi:10.1002/14651858.CD004963.pub3

Joo, N.-S., \& Kim, B.-T. (2007). Mobile phone short message service messaging for behaviour modification in a community-based weight control programme in Korea. Journal of Telemedicine and Telecare, 13(8), 416-20. doi:10.1258/135763307783064331

Kim, H.-S., Choi, W., Baek, E. K., Kim, Y. A., Yang, S. J., Choi, I. Y., \& Cho, J.-H. (2014). Efficacy of the smartphone-based glucose management application stratified by user satisfaction. Diabetes \& Metabolism Journal,38(3), 204-10. doi:10.4093/dmj.2014.38.3.204

Kirwan, M., Duncan, M. J., Vandelanotte, C., \& Mummery, W. K. (2012). Using smartphone technology to monitor physical activity in the 10,000 Steps program: a matched case-control trial. Journal of Medical Internet Research, 14(2), e55. doi:10.2196/jmir.1950

Klasnja, P., \& Pratt, W. (2012). Healthcare in the pocket: mapping the space of mobile-phone health interventions. Journal of Biomedical Informatics, 45(1), 184-98. doi:10.1016/j.jbi.2011.08.017

Kohl, H. W., Craig, C. L., Lambert, E. V., Inoue, S., Alkandari, J. R., Leetongin, G., \& Kahlmeier, S. (2012). The pandemic of physical inactivity: global action for public health. Lancet, 380(9838), 294305. doi:10.1016/S0140-6736(12)60898-8

Krishna, S., Boren, S. A., \& Balas, E. A. (2009). Healthcare via cell phones: a systematic review. Telemedicine Journal and E-Health: The Official Journal of the American Telemedicine Association, 15(3), 231-40. doi:10.1089/tmj.2008.0099

Lamina, S., Okoye, C. G, \& Hanif, S. M. (2014). Effects of interval 
exercise training programme on theindices of adiposity and biomarker of inflammation in hypertension: a randomised controlled trial. The Nigerian Postgraduate Medical Journal, 21(2), 136-43.

Levinger, I., Goodman, C., Matthews, V., Hare, D. L., Jerums, G., Garnham, A., \& Selig, S. (2008). BDNF, metabolic risk factors, and resistance training in middle-aged individuals. Medicine and Science in Sports and Exercise, 40(3), 535-41. doi:10.1249/ MSS.0b013e31815dd057

Logan, A. G, McIsaac, W. J., Tisler, A., Irvine, M. J., Saunders, A., Dunai, A., \& Cafazzo, J. A. (2007). Mobile phone-based remote patient monitoring system for management of hypertension in diabetic patients. American Journal of Hypertension, 20(9), 942-8. doi:10.1016/j.amjhyper.2007.03.020

Martínez, C. S., Ortega, M. V., Párraga Montilla, J. A., \& Moreno, R. (2010). Efectos de la utilización de los videojuegos en un programa de mejora del equilibrio en mujeres de 60 años o más Effects of the use of videogames in a balance improving programme for women of 60 or over. Retos, 17, 93-95.

Mathers, C. D., Stevens, G. a, Boerma, T., White, R. a, \& Tobias, M. I. (2014). Causes of international increases in older age life expectancy. The Lancet, 6736(14), 1-9. doi:10.1016/S0140-6736(14)60569-9

Middelweerd,A., Mollee, J. S., van der Wal, C., Brug, J., \& Te Velde, S. J. (2014). Apps to promote physical activity among adults: a review and content analysis. The International Journal of Behavioral Nutrition and Physical Activity, 11(1), 97. doi:10.1186/s12966-0140097-9

Moreira, S. R., Cucato, G. G., Terra, D. F., \& Ritti-Dias, R. M. (2014). Acute blood pressure changes are related to chronic effects of resistance exercise in medicated hypertensives elderly women. Clinical Physiology and Functional Imaging. doi:10.1111/cpf.12221

Müller, A. M., \& Khoo, S. (2014). Non-face-to-face physical activity interventions in older adults: a systematic review. The International Journal of Behavioral Nutrition and Physical Activity, 11, 35. doi:10.1186/1479-5868-11-35

Nelson, M. E., Rejeski, W. J., Blair, S. N., Duncan, P. W., Judge, J. O., King, A. C., \& Castaneda-Sceppa, C. (2007). Physical activity and public health in older adults: recommendation from the American College of Sports Medicine and the American Heart Association. Medicine and Science in Sports and Exercise, 39(8), 1435-45. doi:10.1249/mss.0b013e3180616aa2

Richards, J., Hillsdon, M., Thorogood, M., \& Foster, C. (2013). Faceto-face interventions for promoting physical activity. The Cochrane Database of Systematic Reviews, 9, CD010392. doi:10.1002 14651858.CD010392.pub2

Richards, J., Thorogood, M., Hillsdon, M., \& Foster, C. (2013). Faceto-face versus remote and web 2.0 interventions for promoting physical activity. The Cochrane Database of Systematic Reviews, 9, CD010393. doi:10.1002/14651858.CD010393.pub2

Rikli, R. E., \& Jones, C. J.(2012). Senior fitness testmanual. Champaign, IL: Human Kinetics (Vol. 1).

Rollo, M. E., Ash, S., Lyons-Wall, P., \& Russell, A. (2011). Trial of a mobile phone method for recording dietary intake in adults with type 2 diabetes: evaluation and implications for future applications. Journal of Telemedicine and Telecare, 17(6), 318-23. doi:10.1258/ jtt.2011.100906

Salinas, F., Cocca, A., Mohamed, K., \& Viciana, J. (2010). Actividad Física y sedentarismo: Repercusiones sobre la salud y calidad de vida de las personas mayores. Retos. Nuevas Tendencias En Educación Física, Deporte Y Recreación, (17), 126-129.

Seto, E., Leonard, K. J., Masino, C., Cafazzo, J. A., Barnsley, J., \& Ross, H. J. (2010). Attitudes of heart failure patients and health care providers towards mobile phone-based remote monitoring. Journal of Medical Internet Research, 12(4), e55. doi:10.2196/ jmir.1627

Severinsen, K., Jakobsen, J. K., Pedersen, A. R., Overgaard, K., \& Andersen, H. (2014). Effects of resistance training and aerobic training on ambulation in chronic stroke. American Journal of
Physical Medicine \& Rehabilitation / Association of Academic Physiatrists, 93(1), 29-42. doi:10.1097/PHM.0b013e3182a518e1

Stephens, J., \&Allen, J. (2013). Mobile phone interventions to increase physical activity and reduce weight: a systematic review. The Journal of Cardiovascular Nursing, 28(4), 320-9. doi:10.1097/ JCN.0b013e318250a3e7

Stuckey, M. I., Kiviniemi, A. M., \& Petrella, R. J. (2013). Diabetes and technology for increased activity study: the effects of exercise and technology on heart rate variability and metabolic syndrome risk factors. Frontiers in Endocrinology, 4(September), 121. doi:10.3389/ fendo.2013.00121

Stuckey, M., Sc, M., Russell-minda, E., Read, E., Munoz, C., Shoemaker, K., \& Petrella, R. (2011). Diabetes and Technology for Increased Activity (DaTA) Study: Results of a Remote Monitoring Intervention for Prevention of Metabolic Syndrome, 5(4), 928935.

Sun, F., Norman, I. J., \& While, A. E. (2013). Physical activity in older people: a systematic review. BMC Public Health, 13, 449. doi:10.1186/1471-2458-13-449

Swift, D. L., Earnest, C. P., Katzmarzyk, P. T., Rankinen, T., Blair, S. N., \& Church, T. S. (2012). The effect of different doses of aerobic exercise training on exercise blood pressure in overweight and obese postmenopausal women. Menopause (New York, N.Y.), 19(5), 5039. doi:10.1097/gme.0b013e318238ea66

Van Dantzig, S., Geleijnse, G., \& van Halteren, A. T. (2012). Toward a persuasive mobile application to reduce sedentary behavior. Personal and Ubiquitous Computing, 17(6), 1237-1246. doi:10.1007/ s00779-012-0588-0

Wang, C.-H., Chou, P.-C., Joa, W.-C., Chen, L.-F., Sheng, T.-F., Ho, S.C., \& Kuo, H.-P. (2014). Mobile-phone-based home exercise training program decreases systemic inflammation in COPD: a pilot study. BMC Pulmonary Medicine, 14, 142. doi:10.1186/1471-2466-14142

Williams, V., Price, J., Hardinge, M., Tarassenko, L., \& Farmer, A. (2014). Using a mobile health application to support selfmanagement in COPD: a qualitative study. The British Journal of General Practice: The Journal of the Royal College of General Practitioners, 64(624), e392-400. doi:10.3399/bjgp14X680473

Yasuda, T., Fukumura, K., Fukuda, T., Iida, H., Imuta, H., Sato, Y., \& Nakajima, T.(2014). Effects of low-intensity, elastic band resistance exercise combined with blood flow restriction on muscle activation. Scandinavian Journal of Medicine \& Science in Sports, 24(1), 5561. doi:10.1111/j.1600-0838.2012.01489.x

Yu, W., An, C., \& Kang, H. (2013). Effects of Resistance Exercise Using Thera-band on Balance of Elderly Adults: ARandomized Controlled Trial. Journal of Physical Therapy Science, 25(11), 1471-3. doi:10.1589/jpts.25.1471

Zheng, L., Sun, Z., Li, J., Zhang, R., Zhang, X., Liu, S., \& Sun, Y.(2008). Pulse pressure and mean arterial pressure in relation to ischemic stroke among patients with uncontrolled hypertension in rural areas of China. Stroke; a Journal of Cerebral Circulation, 39(7), 1932-7. doi:10.1161/STROKEAHA.107.510677

Zion, A. S., De Meersman, R., Diamond, B. E., \& Bloomfield, D. M. (2003). Ahome-based resistance-training program using elastic bands for elderly patients with orthostatic hypotension. Clinical Autonomic Research: Official Journal of the Clinical Autonomic Research Society, 13(4), 286-92. doi:10.1007/s10286-003-0117-3

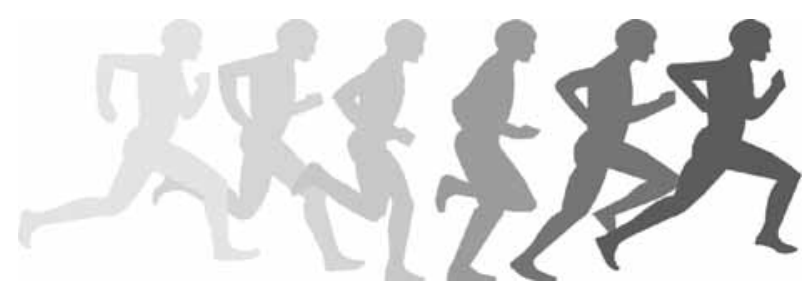

